\title{
Oshidori-Net: Connecting Regional EPR Systems to Achieve Secure Mutual Reference with Thin-Client Computing Technology
}

\author{
Shigeki Kuwata ${ }^{1}$, Koji Toda ${ }^{2}$, Kei Teramoto ${ }^{1}$, Hiroshi Kondoh ${ }^{1}$, \\ Hiroshige Nakamura ${ }^{1}$, Hirohiko Murata ${ }^{2}$, Noriaki Tamura ${ }^{2}$, and Masahide Ikeguchi ${ }^{1}$ \\ ${ }^{1}$ Tottori University Hospital, \\ 36-1 Nishi-cho, Yonago, Tottori, 683-8504, Japan \\ ${ }^{2}$ Saihaku Municipal Hospital, \\ 397 Yamato, Nanbu, Saihaku, Tottori, 683-0323, Japan \\ \{shig, kei, kondoh, hnaka, masaike\} @med. tottori-u.ac.jp, \\ \{toda.k, tamura.n\} atown. nanbu.tottori.jp
}

\begin{abstract}
A mutual reference system developed by the authors, Oshidori-Net, facilitated medical professionals to remotely access to the existing electronic patient record systems. The system was technically oriented based on the thinclient technology in terms of security enhancement and cost reduction. Formerly a small portion of the patient healthcare information was provided for the counterpart, whereas, by introducing the system, all the electronically formatted information stored in the systems was shared with the professionals so that reduction of duplicated health examinations and improvement of healthcare service in both hospitals were anticipated. The regional medical association with the system would form an ideal model in next generation to achieve virtual full-functioned hospitals providing the best quality of medical services.
\end{abstract}

Keywords: Electronic Patient Records, Thin-client Computing, Regional Healthcare Service.

\section{Introduction}

A Newly developed service, Oshidori-Net, providing medical workers with remote access to electronic patient record systems (EPR) of regional hospitals mutually connected using the thin-client computing technology, has been in operation with the participation of a university-affiliated hospital with 697 beds (Tottori University Hospital; TUH) and a regional medical center with 198 beds (Saihaku Municipal Hospital; SMH) in a rural district of Japan since July, 2009. The system was designed based on the thin-client computing (TCC) architecture that TUH had adopted for its intranet system [1] [2], achieving higher security at a low cost with the least modification to the existing hospital information systems. 


\subsection{Introduction of Thin-Client Computing Technology in Tottori University Hospital}

TUH introduced physician's order entry and EPR to the hospital information system in 2003. As approximately 900 medical specialists with 1,000 client PCs at TUH had used the system for over five years, they were gradually dependant upon the system environment and considered it to be an essential infrastructure for their daily work. The trend caused a growing need of many client PCs by the users: they needed workstation PCs to input orders and to check the results of tests wherever they worked. In response to the requirement, it was of urgent necessity for information managers of the hospital to supply additional client PCs without increasing the risk related to cost and security. TUH introduced models of thin-client computing to the system, also known as server-based computing (SBC), to solve the problem [1].

\subsection{Potentiality and Effectiveness of the Thin-Client Computing Technology}

As a characteristic of TCC technology, middleware servers (TCC servers) installed between PC clients and database servers of the system are able to process multiple users' applications and to behave themselves as virtual clients providing each PC client with only their screen information without transferring real data (Fig.1).
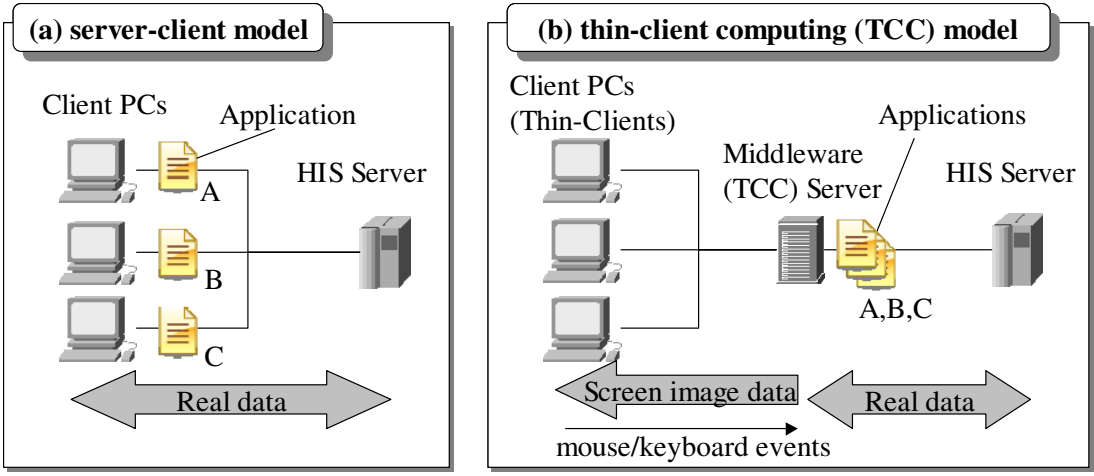

Fig. 1. Difference on a system configuration and a data transferring method between (a) a server-client model and (b) a thin-client computing (TCC) model [1]

This technology drew an attention of hospital information managers in term of its superiority with regard to cost reduction and privacy protection, accordingly its implementation to the hospital information system was carried out in several medical facilities though its application tended to be limited to relatively small domains, e.g., medical telemetry for monitoring sleep-related disorders [3], remote access to the laboratory system [4] and simulation systems for dentistry [5].

As assistance for implementing TCC in large-scale facilities, the authors developed a design model under real hospital settings [2]. In the major replacement of the hospital information system at TUH in 2008, TCC was implemented according to the design model [6]. Consequently about 1,100 client PCs in cooperation with 70 middleware TCC 
servers worked successfully on the TCC system. The implementation was the first attempt in large hospital settings in Japan.

The authors also evaluated the effectiveness of the system after the implementation. The introduction of thin-clients contributed to the reduction of PC failure rates: the hospital logged 39 failures in the past ten month, while 77 failure cases occurred per year before the implementation. Electric energy consumption was also reduced: the electric power consumed by client PCs averaged $40.5 \mathrm{KW}$, while that by TCC servers and client PCs averaged 36.1 KW for the new system [1].

\section{Purpose of the System}

Oshidori-Net allowed medical professionals working for the hospitals to refer to the existing EPRs (CIS-MR by IBM Corp. [7] at TUH and HOPE/EGMAIN-FX by Fujitsu Limited [8] at SMH) for healthcare information of patients who had consented to the mutual referral by the professionals. Formerly a small portion of the patient healthcare information, e.g., referral letters with X-ray films, was provided for the counterpart, whereas, by introducing the system, all the electronically-formatted information stored in the systems was shared with the professionals so that reduction of duplicated health examinations and improvement of healthcare service in both hospitals were anticipated. In addition, medical resources in the university hospital, such as skilled specialists or advanced devices, could be remotely utilized by the general hospital to form the virtual full-functioned medical center in the region.

\section{System Design}

The thin-client computing system at TUH (GO-Global by GraphOn Corp. [9]) was successfully extended to the network system of the counterpart via the Tottori Information Highway, a closed wide-area giga-bit network in Tottori Prefecture region (Fig.2). Firewalls (Cisco 1812J with Cisco IOS Firewall) were installed at the connection node of the network in both hospitals to form a shared-network domain (namely DMZ) and protect the intranet where the patient healthcare information was stored. Desktop virtualization servers (DVSs), as TCC servers providing the thinclient computing, were installed in the shared-network to play a role as proxy servers to retrieve the information and transfer it to remote clients. The specification of DVSs was composed of a single CPU of Quad Core X-E5405 with a clock frequency of $2 \mathrm{GHz}$, 5GB RAM, four 36GB-HDDs with RAID-5 configuration having a hot spare disk and Windows Server Standard 2003 operating system (Hewlett Packard ProLiant DL360 G5). Thin-client computing middleware, GO-Global, was installed in the DVSs to make every application for EPR and PACS run on the DVSs for multiple login users, with its screen information compressed, encrypted and transferred to the client PCs of the users by the Rapid-X protocol over TCP/IP network. The designed architecture yielded great advantage of the security aspects that frequently aroused concern in people involved; the patient healthcare information transferred to the users was composed of a series of screen images without containing its original data. The innovative architecture was able to minimize the risk that an enormous amount of data 
might be divulged in a very short moment. In an attempt to further enhance the security protection, use of printers and clipboard functions on DVSs was not allowed to the remote users.

To maintain stable and secure system operation, roles of the system administrators were defined as follows: (1) to conduct a mutual audit on the system utilization of the counterpart users, and (2) to provide an instruction course with the users on privacy protection, precedent to the permission granted to the users.

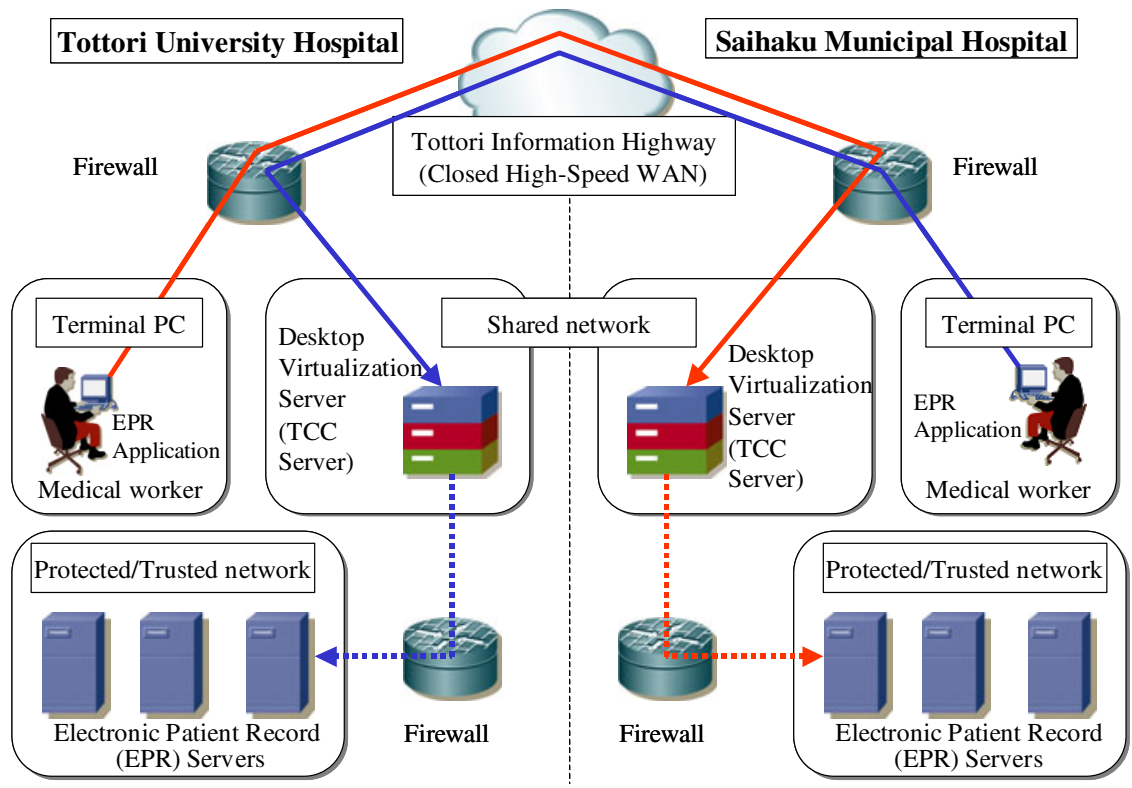

Fig. 2. Conceptual system configuration model of the electronic patient record systems connected with thin-client technology to achieve secure mutual reference

\section{Operational Workflow of the System}

\subsection{User Registration}

All users in TUH and SMH were qualified to use the system after they completed an educational course on the system operation and its security provided upon registration. The course was provided by a system administrator at TUH in a classroom style on a regular basis and an e-learning (online) style available anytime on the Internet. User registration was conducted manually and asynchronously at two hospitals: When new users were registered or any attributes (e.g. name, position and department) on existent users were altered at one hospital, the system administrator of the hospital sent the users' information to a system administrator of the other hospital in some secure ways (via facsimile or via encrypted email transmission), followed by the user registration at the latter hospital. 


\subsection{Patient Registration (Fig.3)}

An informed consent form should be submitted to one of the hospitals prior to the patient registration into the system. The consent form was first sent to an office administrator of the hospital, to which the patient submitted the form, subsequently to a system administrator to grant read-only permission of the patient information in EPR from the other hospital. The permission would last up to three years without exceptional circumstances. Users of the other hospital were only able to refer to the information of the patients with the permission. Upon completion of the registration, the form was stored in a department of the hospital (e.g. health information management center) according to rules decided by the hospital. The office administrator sent the patient's information to an office administrator of the other hospital via facsimile, followed by the same procedures at the latter hospital.

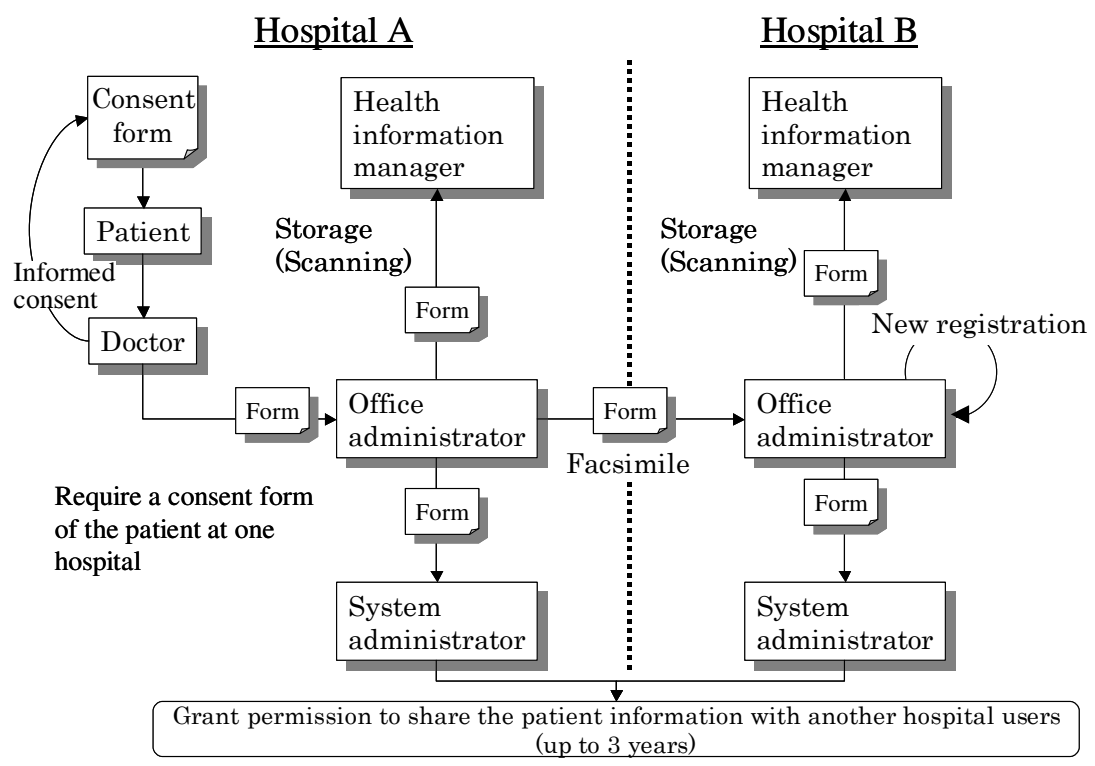

Fig. 3. Workflow of patient registration in Oshidori-net

\section{Results}

As of January 20, 2010, 66 patients and 245 medical professionals, including 194 for TUH 51 for SMH, were registered for the mutual reference system. The frequency of the patient registration, compared with the observed patient referrals in 2008, corresponded to approximately a half of all the referrals.

Fig.4 shows the frequency of data access to the counterpart system. The fact that access from SMH to TUH was considerably dominant was caused by the imbalance of the need requiring the patient information between the hospitals: TUH as a university hospital tended to provide more advanced medical service than SMH 
resulting in a growing need of the professionals at SMH for the information unavailable on site. On the other hand, the doctors at TUH tended to retrieve the information stored at SMH only upon referral.

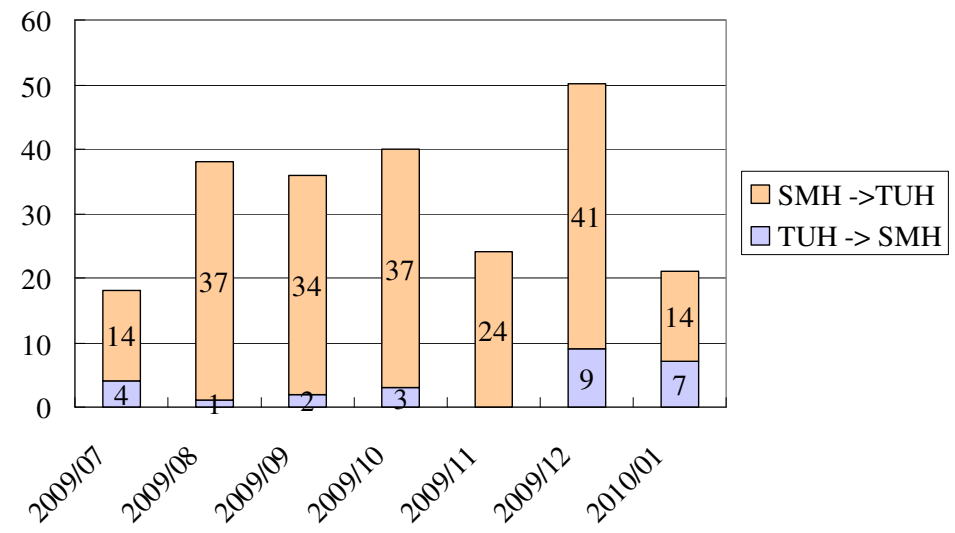

Fig. 4. Frequency of data access to the counterpart system. Consecutive transactions with its duration within 20 minutes were counted once.

SMH: Saihaku Municipal Hospital, TUH: Tottori University Hospital.

Frequency of referred information stored at TUH was presented in Fig.5. The most frequent reference occurred in progress note $(37 \%)$, followed by blood test $(7 \%)$, radiology image $(5 \%)$, prescription $(4 \%)$ and so forth. Preliminary brief interviews with the doctors at $\mathrm{SMH}$ revealed their affirmative impression that the system facilitated the access to the detailed information to know the background or progress on the patient treatment.

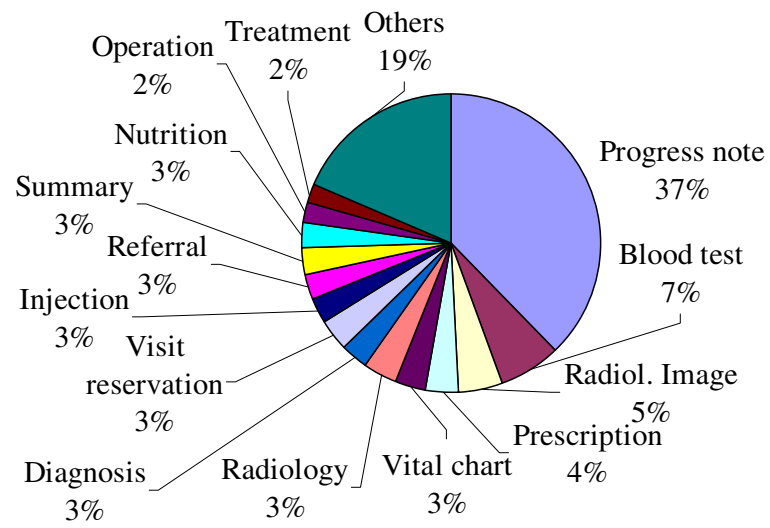

Fig. 5. Ratios of frequency of data access from Saihaku Municipal Hospital (SMH) to Tottori University Hospital (TUH) grouped by sorts of referenced data.

Consecutive transactions to the same referred information with its duration within 10 minutes were counted once. The counted transactions from SMH to TUH were 528 in total. 


\section{Discussion}

Introduction of the system has altered the way of referrals between the hospitals; formerly portable media, e.g., CD or DVD, were attached to paper-based referral letters to send massive DICOM images and accompanied reports, whereas at present only brief messages are exchanged upon referral. In the conventional offline method, provided DICOM images are often copied to the storage server again at the counterpart resulting in excessive redundancy of the images among regional hospitals leading to the unsolicited increase of cost. The system presents an efficient solution for the issue with practical tactics. An analogous instance was found in a large-scale system, Ajisai-net [10] [11], that consisted of approximately 10,000 patients and more than 120 hospitals registered at a relatively low cost by partly using thin-client computing architecture.

Detail description of patients on referrals was also of great need to doctors as illustrated in Fig.5 in which progress notes were by far the most frequently viewed resources. The result implied that detail information in progress notes would be utilized effectively on referrals in addition to DICOM images and discharge summaries that were often transferred with portable media. Though the necessity of such information has been recognized so far, delays in establishing standards would have reduced the possibility to exchange such complicatedly structured and often nonstructured information in progress notes. The system, Oshidori-net, simply provided mutual reference of all information in EPR for users, but it would be one of effective solutions in practical use for medical professionals until applicable and feasible standards become widespread for medical data exchange between hospitals.

Some issues were still to be solved in further development of the system. One instance was that professionals registered in the system, specifically doctors, often insisted that the system be more convenient for the users, even allowing for some violations of the policies for privacy protection (e.g. capturing screen information of the other hospital's EMR to duplicate the data which would lead to privacy infringement). The demand obviously stemmed from the strict security implementation enforced in the system operation, therefore the modification would be considered in terms of the usability enhancement. Another issue was on scalability of the system that would be required, as the participation of hospitals would be increased. Some features of the IHE technical frameworks, e.g., PIX or XUA, should be incorporated into the system in further development to maintain the operability and consistency of the system.

\section{Conclusion}

The mutual reference system developed by the authors facilitated medical professionals to access to the EMR data from a remote site. The system was technically oriented based on the thin-client technology in terms of security enhancement and cost reduction. The regional medical association with the system would form an ideal model in next generation to achieve the advanced virtual hospital providing the best quality of medical services. 


\section{References}

1. Kuwata, S., Teramoto, K., Matsumura, Y., Kushniruk, A.W., Borycki, E.M., Kondoh, H.: Effective solutions in introducing server-based computing into hospital information system. Stud. Health. Technol. Inform. 143, 435-440 (2009)

2. Teramoto, K., Kuwata, S., Nishimura, M., Kumagai, T., Saigo, T., Kondoh, H.: Development of a design model of Hospital Information System based on Server-Based Computing. Japan Journal of Medical Informatics 27(suppl.), 666-668 (2007)

3. Astaras, A., Arvanitidou, M., Chouvarda, I., Kilintzis, V., Koutkias, V., Sanchez, E.M., et al.: An integrated biomedical telemetry system for sleep monitoring employing a portable body area network of sensors (SENSATION). In: Conf. Proc. IEEE Eng. Med. Biol. Soc., pp. 5254-5257 (2008)

4. Ulma, W., Schlabach, D.M.: Technical Considerations in Remote LIMS Access via the World Wide Web. J. Autom. Methods Manag. Chem., 217-222 (2005)

5. Taylor, D., Valenza, J.A., Spence, J.M., Baber, R.H.: Integrating electronic patient records into a multi-media clinic-based simulation center using a PC blade platform: a foundation for a new pedagogy in dentistry. In: AMIA Annu. Symp. Proc., pp. 11-29 (2007)

6. Teramoto, K., Kuwata, S., Kawai, T., Imai, Y., Fujii, K., Nishimura, M., Kondo, H.: Evaluation of a design model of Hospital Information System based on Server-Based Computing (SBC). Japan Journal of Medical Informatics 28(suppl.), 376-379 (2008)

7. CIS-MR (Medical Record), IBM Corporation (in Japanese), http: / / www-06.ibm.com/industries/jp/heal thcare/ solution/cismr.html (accessed May 1, 2010)

8. HOPE/EGMAIN-FX, Fujitsu Limited (in Japanese), http://segroup.fujitsu.com/medical/products/egmainfx (accessed May 1, 2010)

9. Graph on Corporation, http: / /www. graphon.com (accessed May 1, 2010)

10. Matsumoto, T., Honda, M.: The actual status and problems of the development of IT in community medicine Ajisai network at Nagasaki. Japan Journal of Medical Informatics 27(suppl.), 164-165 (2007)

11. Kimura, H., Nakahara, K., Yonekura, M.: The operative experience of five years of the regional medicine cooperation system (Ajisai net). Japan Journal of Medical Informatics 29(suppl.), 530-531 (2009) 